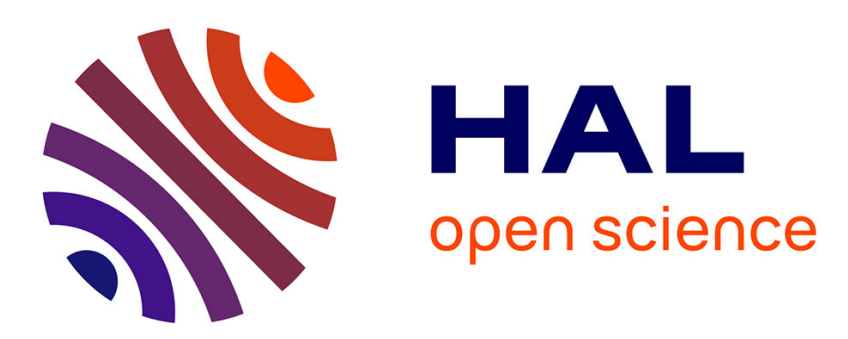

\title{
Contact angle hysteresis and pinning at periodic defects in statics
}

\author{
Stanimir S Iliev, Nina S Pesheva, Vadim Nikolayev
}

\section{To cite this version:}

Stanimir S Iliev, Nina S Pesheva, Vadim Nikolayev. Contact angle hysteresis and pinning at periodic defects in statics. Physical Review E: Statistical, Nonlinear, and Soft Matter Physics, 2014, 90, pp.12406. 10.1103/PhysRevE.90.012406 . cea-01384214

\section{HAL Id: cea-01384214 https://hal-cea.archives-ouvertes.fr/cea-01384214}

Submitted on 19 Oct 2016

HAL is a multi-disciplinary open access archive for the deposit and dissemination of scientific research documents, whether they are published or not. The documents may come from teaching and research institutions in France or abroad, or from public or private research centers.
L'archive ouverte pluridisciplinaire HAL, est destinée au dépôt et à la diffusion de documents scientifiques de niveau recherche, publiés ou non, émanant des établissements d'enseignement et de recherche français ou étrangers, des laboratoires publics ou privés. 


\title{
Contact angle hysteresis and pinning at periodic defects in statics
}

\author{
Stanimir Iliev, ${ }^{1, *}$ Nina Pesheva, ${ }^{1, \dagger}$ and Vadim S. Nikolayev ${ }^{2, \dagger}$ \\ ${ }^{1}$ Institute of Mechanics, Bulgarian Academy of Sciences, Academician Georgi Bonchev Street 4, 1113 Sofia, Bulgaria \\ ${ }^{2}$ SPEC/SPHYNX (CNRS URA 2464), DSM/IRAMIS CEA Saclay, 91191 Gif-sur-Yvette, France \\ (Received 4 November 2013; revised manuscript received 21 May 2014; published 31 July 2014)
}

\begin{abstract}
This article deals with the theoretical prediction of the wetting hysteresis on nonideal solid surfaces in terms of the surface heterogeneity parameters. The spatially periodical chemical heterogeneity is considered. We propose precise definitions for both the advancing and the receding contact angles for the Wilhelmy plate geometry. It is well known that in such a system, a multitude of metastable states of the liquid meniscus occurs for each different relative position of the defect pattern on the plate with respect to the liquid level. As usual, the static advancing and receding angles are assumed to be a consequence of the preceding contact line motion in the respective direction. It is shown how to select the appropriate states among all metastable states. Their selection is discussed. The proposed definitions are applicable to both the static and the dynamic contact angles on heterogeneous surfaces. The static advancing and receding angles are calculated for two examples of periodic heterogeneity patterns with sharp borders: the horizontal alternating stripes of a different wettability (studied analytically) and the doubly periodic pattern of circular defects on a homogeneous base (studied numerically). The wetting hysteresis is determined as a function of the defect density and the spatial period. A comparison with the existing results is carried out.
\end{abstract}

DOI: 10.1103/PhysRevE.90.012406

PACS number(s): 68.03.Cd, 05.90.+m, 68.08.Bc

\section{INTRODUCTION}

There are yet many open questions in the wetting statics and dynamics [1] in spite of the strong interest in these topics by both the scientific community and industry. One of the open issues concerns the wetting on heterogeneous and/or rough solid surfaces. The surface heterogeneities that we call hereafter "defects" are well known to be responsible for the stick-slip dynamics of the contact line (CL) and the nonuniqueness of the static contact angle (CA), which is a phenomenon responsible for the appearance of the wetting hysteresis. It is ubiquitous for all real surfaces [2] and is thus an important problem.

The main objective of the theoretical description of wetting is a prediction of behavior on macroscopic length scale (e.g., the average meniscus curvature or drop size) in terms of the heterogeneity parameters, defined on a much smaller length scale.

The Cassie-Baxter relation for the averaged cosine of the static CA was one of the first attempts of such an averaging. However, the introduction of this unique averaged CA is not sufficient for characterizing heterogeneous surfaces due to the existence of wetting hysteresis. One needs to define other parameters to characterize the wetting on a heterogeneous and/or rough surfaces. The static advancing $\theta^{a}$ and receding $\theta^{r}$ contact angles (ACA and RCA) are used for this purpose. However, the definitions in the literature for the wetting hysteresis are not consistent (see Ref. [3], and references therein) and lead to ambiguous results.

The appearance of different values of the CA is related to the existence of both a multitude of metastable states of the liquid meniscus for a given position of the defect pattern on

\footnotetext{
*stani@imbm.bas.bg

†nina@imbm.bas.bg

${ }^{\ddagger}$ vadim.nikolayev@cea.fr
}

the plate with respect to the liquid and the different possible positions of the defect pattern on the plate with respect to the liquid, over which the averaging should be performed. A wetting hysteresis theory should give answers to the following questions: (i) how to determine the CA distribution along the CL for a specific system realization, (ii) how to select the advancing and receding CAs for the specific system realization from the set of all possible values of averaged CAs, (iii) how to choose from different suggested ways of averaging the CA (see Sec. III A), and (iv) how to average the CAs over the surface, due to the existence of many different possible relative positions of the defect pattern on the plate with respect to the liquid meniscus. The answer to these questions is not unique and there is still an ongoing discussion. Further work is needed to elucidate these problems.

An effort towards a more consistent theoretical description of hysteresis, i.e., definitions and clear procedure for their application is thus necessary. The objective of this study is to suggest precise expressions for these angles that can be used in both the static and the dynamic (for small capillary and Reynolds numbers) cases. We limit ourselves here to the study of chemical heterogeneity in statics; however, the approach, we use here is more general and is applicable for studying the system in contact with arbitrary heterogeneous and/or rough surfaces.

A large number of studies of CA hysteresis on heterogeneous surfaces exist. The hysteresis was calculated for different regular and random patterns of the solid surface by the continuum media energy minimization (see, e.g., [4-10]), lattice-Boltzmann method [11], phase-field models [12], and molecular dynamics approaches [13], to mention just a few.

We focus here on the Wilhelmy balance geometry (Fig. 1) used conventionally for the CA determination. The static ACA measurement in such a system may be performed by fixing the vertical position of the defect pattern on the plate with respect to the liquid level far from the plate, after having moved the plate downwards into the liquid pool. Similarly, the 


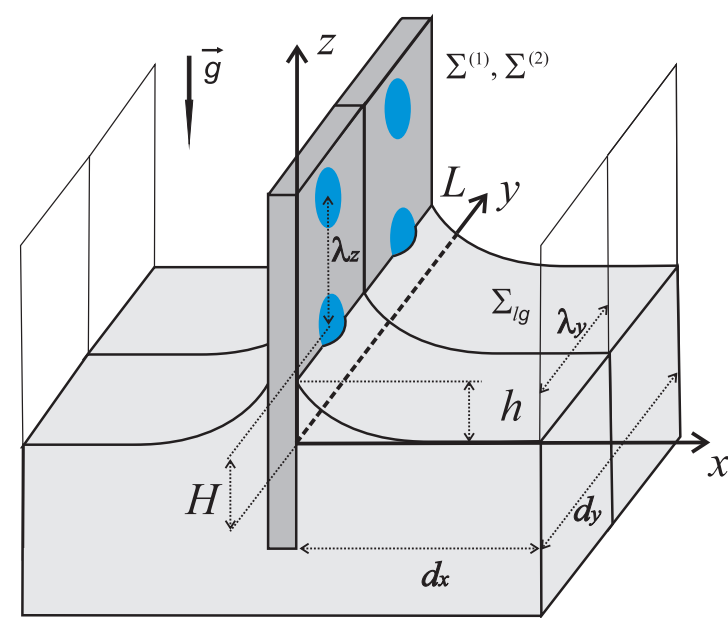

FIG. 1. (Color online) Schematic drawing of the Wilhelmy plate geometry. The vertical plate has surface defects of different wettability, shown with darker color.

RCA may be obtained after the upward motion. The different possible relative positions of the defect pattern on the plate with respect to the liquid level far from the plate (see Fig. 1) are characterized by the values of a parameter $H$ (detailed further in the text). For each $H$, there is a variety of metastable meniscus shapes (that we call states) that may be characterized by the CL height $\langle h\rangle$ (cf. Fig. 1), averaged along the CL (the angle brackets are used hereafter to denote such a "horizontal" averaging). It is well known [4,14-16] that among them the most important for the hysteresis determination are the two states with the highest and the lowest possible values of $\langle h\rangle$, beyond which no metastable meniscus state exists. We denote them with the "+" and "-" superscripts, respectively, so that the metastable states exist for $\left\langle h^{-}\right\rangle \leqslant\langle h\rangle \leqslant\left\langle h^{+}\right\rangle$. The ACA is related to the "-" state, while RCA is defined by the "+" state. These states are the most important for the determination of the CA hysteresis.

The derived RCA and ACA expressions are then applied to two examples of heterogeneous pattern. For the first of them, the horizontal alternating stripes of a different wettability, the hysteresis is obtained analytically. For the second, the doubly periodic pattern of circular defects on a homogeneous base, two methods are used and their results compared: the direct numerical simulation method and the semianalytical small slope approximation. We compare our results with the existing hysteresis determination methods (in particular, with [17]) and consider the wetting hysteresis dependence on the concentration of periodically distributed defects.

\section{PROBLEM FORMULATION}

A flat heterogeneous solid plate is immersed vertically into a liquid pool. The plate vertical position may be varied, so that the defect pattern position $H$ with respect to the liquid level in the pool is controlled. One of the plate faces (we do not consider the other) is described with the Cartesian coordinates $(y, z)$ where the $y$ axis is horizontal and the $z$ axis is directed upward, as shown in Fig. 1, with zero coinciding with the liquid level far from the moving plate. The liquid free surface is denoted by $\Sigma_{l g}$ and its surface tension by $\gamma_{l g}$. The CL, which the liquid meniscus forms with the solid surface, is denoted by $L$ and its height by $h$. We consider here the case when the free liquid surface $\Sigma_{l g}$ is a single-valued function of $x \geqslant 0$ and $y: z=f(x, y) ; h=f(0, y)$. A CL point is then described by the vector $\vec{R}=[y, h]$. The distance between the plate and the container wall, opposite to the plate, is denoted by $d_{x}$, where $d_{x} \gg l_{c}\left[l_{c}=\sqrt{\gamma_{l g} /\left(\rho_{l}-\rho_{g}\right) g}\right.$ is the capillary length; the density of the liquid and of the ambient gas are denoted by $\rho_{l}$ and $\rho_{g}$, respectively; $g$ is the gravity acceleration]. The width of the plate is denoted by $d_{y}$.

In the past, many studies (see, e.g., [9]) used the approach of Joanny and de Gennes [18] developed for smooth variation of solid surface energy. We focus here on the case on the "mesatype" defects when the plate surface $\Sigma$ consists of two kinds of domains $\Sigma^{(i)}(i=1,2)$ of different wettability with sharp borders between them. It is assumed that both domains are homogeneous, with no intrinsic wetting hysteresis. Inside the domain $\Sigma^{(i)}$, the solid-liquid interface energy $\gamma_{s l}(y, z)=\gamma_{s l}^{(i)}$ and the solid-gas surface energy $\gamma_{s g}(y, z)=\gamma_{s g}^{(i)}$. These surface energies correspond to the Young CAs,

$$
\cos \theta_{\mathrm{eq}}^{(i)}=\left(\gamma_{s g}^{(i)}-\gamma_{s l}^{(i)}\right) / \gamma_{l g} .
$$

Without any loss of generality, it is assumed in the following that

$$
\theta_{\text {eq }}^{(1)}<\theta_{\text {eq }}^{(2)} \text {. }
$$

The domain pattern is assumed to be periodical, with spatial periods $\lambda_{y}$ and $\lambda_{z}$ in $y$ and $z$ directions, respectively (Fig. 1). It is assumed that $d_{y} \gg \lambda_{y}$.

Here we concentrate on the case of mesoscopic scale heterogeneities. Because of their large length scale, thermal effects [1] are not expected to influence the hysteresis so that the zero-temperature case may be considered. The free energy $U$ of the system is thus [19]

$$
\begin{aligned}
U= & \int_{\Sigma_{l g}} \gamma_{l g} d \Sigma+\int_{\Sigma_{s l}} \gamma_{s l} d \Sigma+\int_{\Sigma_{s g}} \gamma_{s g} d \Sigma \\
& +\int_{\Omega_{l}} \rho_{l} g z d \Omega+\int_{\Omega_{g}} \rho_{g} g z d \Omega,
\end{aligned}
$$

where the liquid and gas volumes are denoted $\Omega_{l}$ and $\Omega_{g}$, respectively. For a given choice of the surface pattern, the free energy (3) is uniquely determined by the position of the liquid meniscus and thus $U$ is a functional of $f(x, y)$. The meniscus metastable states (including those related to the advancing and receding contact angles) correspond to the local minima of the free energy (3). The minimization should thus be performed to answer the question (i) posed in Sec. I.

For mesa type defects, the minimization leads [6] to the following conditions for the equilibrium local CA $\theta(\vec{R})$, which is the meniscus slope at $x=0$. For the points $\vec{R} \in L$ that belong to the interiors of domains, the following condition is valid:

$$
\theta(\vec{R})=\left\{\begin{array}{lll}
\theta_{\mathrm{eq}}^{(1)}, & \text { if } & \vec{R} \in \Sigma^{(1)}, \\
\theta_{\mathrm{eq}}^{(2)}, & \text { if } & \vec{R} \in \Sigma^{(2)}
\end{array}\right.
$$


For the points $\vec{R}_{b} \in L$ at the borders between the domains, the following inequality should be satisfied

$$
\theta_{\mathrm{eq}}^{(1)} \leqslant \theta\left(\vec{R}_{b}\right) \leqslant \theta_{\mathrm{eq}}^{(2)} .
$$

In other words, when the CL attains a point $\vec{R}_{b}$ at a border between the domains, the CL remains pinned as long as the local CA satisfies the double inequality (5). In this case the equilibrium may occur only when the liquid situates at the side of the defect border, corresponding to the domain with the better wettability (domain 1 ).

\section{DEFINITIONS OF ADVANCING AND RECEDING CONTACT ANGLES}

Consider now the questions (iii) and (iv) of Sec. I, i.e., the CA averaging over the solid surface. It is more convenient to discuss separately the averaging over the horizontal $(y)$ and the vertical $(z)$ directions; cf. Fig. 1.

\section{A. Horizontal averaging}

After an equilibrium meniscus state is found for a fixed plate position, one can define the averaged CA. There are many possible ways to define averaged angles. One can, e.g., introduce the angle averaged along the CL $L$ (or along the width of the plate), $\vec{R} \in L$,

$$
\frac{1}{|L|} \int_{L} \theta(\vec{R}) d l
$$

where $|L|$ is the CL length.

Other definitions of average angle have been also considered by different researchers. For example, a projection angle is introduced in [6] and an apparent angle is defined in [20] (see also [1]). Such definitions are basically similar to that of David and Neumann [9], who define the average angle $\langle\theta\rangle$ by the expression

$$
\langle\theta\rangle=\arcsin \left(1-\frac{\langle h\rangle^{2}}{2 l_{c}^{2}}\right),
$$

deduced from the equilibrium averaged over $y$ CL height $\langle h\rangle$ via the well known relation between the CA $\theta$ and the CL height $h$ on an ideally homogeneous wall [19]:

$$
h=l_{c} \sqrt{2(1-\sin \theta)} .
$$

In the present study, we adopt another definition of averaging the $\mathrm{CA}$, related to the values of the RCA and ACA, measured experimentally by the Wilhelmy balance method [21,22]. The average angle is deduced from the vertical component of the force, exerted by the liquid on the Wilhelmy plate, more specifically, from its "capillary" part, related to the presence of the CL. Note that we consider only one side of the plate (Fig. 1), while the force is exerted on both sides. The force per unit length of the CL $L$ is $\gamma_{l g} \cos \theta(\vec{R}) \vec{m}(\vec{R})$ [23], where the unit vector $\vec{m}(\vec{R})$, defined at $\vec{R} \in L$, is normal to the CL $L$, is tangent to the liquid-solid interface, and points outward from the liquid. The $z$ component of the total force per unit width of the plate is thus $[10,22]$

$$
F=\frac{\gamma_{l g}}{|L|} \int_{L} \cos \theta(\vec{R}) \vec{m}(\vec{R}) \cdot \vec{z} d l,
$$

where $\vec{z}$ is the unit vector directed along the $z$ axis. A change of variables allows us to write this as

$$
F=\frac{\gamma_{l g}}{d_{y}} \int_{0}^{d_{y}} \cos \theta(y, h(y)) d y .
$$

It is evident now that the ratio $F / \gamma_{l g}$ defines the cosine of the CA averaged over $y$ :

$$
\langle\cos \theta\rangle=\frac{1}{d_{y}} \int_{0}^{d_{y}} \cos \theta(y, h(y)) d y .
$$

The average angle is obtained as the inverse cosine of this quantity. For the case of periodical defects, $\theta$ and $h$ are periodical functions of $y$ and it is sufficient to average over one period $\lambda_{y}$,

$$
\langle\cos \theta\rangle=\frac{1}{\lambda_{y}} \int_{0}^{\lambda_{y}} \cos \theta(y, h(y)) d y .
$$

The CA hysteresis definition [1] as a difference of the cosines of the advancing and receding CAs gives one more reason to average the cosine of the local CAs and not the CAs themselves.

\section{B. Vertical averaging}

For a pattern whose wettability varies in the vertical direction, the " \pm " states that are crucial for the wetting hysteresis determination depend also on the relative position $H$ (in the vertical direction) of the heterogeneity pattern with respect to the liquid level. Therefore, to obtain the wetting hysteresis in this case one needs to consider also the averaging along the vertical direction.

For spatially periodical patterns, the CL heights $h^{+}$and $h^{-}$ are periodical functions not only of $y$ but also of $H ; h^{ \pm}(y, H+$ $\left.\lambda_{z}\right)=h^{ \pm}(y, H)$ for any $H$, where $\lambda_{z}$ is the vertical period of the defect pattern. The meniscus states and the corresponding CLs thus need to be determined only for $H \in\left[0, \lambda_{z}\right)$.

For the CLs with $h^{ \pm}=h^{ \pm}(y, H)$, one can calculate the corresponding CA values and their horizontally averaged cosines,

$$
\begin{aligned}
& \langle\cos \theta\rangle^{+}=\frac{1}{\lambda_{y}} \int_{0}^{\lambda_{y}} \cos \theta\left(y, h^{+}(y, H)\right) d y, \\
& \langle\cos \theta\rangle^{-}=\frac{1}{\lambda_{y}} \int_{0}^{\lambda_{y}} \cos \theta\left(y, h^{-}(y, H)\right) d y .
\end{aligned}
$$

It is evident that $\langle\cos \theta\rangle^{-}$and $\langle\cos \theta\rangle^{+}$are the upper and the lower bounds of $\langle\cos \theta\rangle$, respectively. They can be used to obtain the advancing and receding CAs [4]. One yet needs to find out how. The most straightforward choices [4] that provide the upper and lower bounds for $\theta(y, z)$ are

$$
\begin{aligned}
\cos \theta^{r, *} & =\min _{0 \leqslant H<\lambda_{z}}\langle\cos \theta\rangle^{+}, \\
\cos \theta^{a, *} & =\max _{0 \leqslant H<\lambda_{z}}\langle\cos \theta\rangle^{-} .
\end{aligned}
$$

Apart from (13), in Schwartz and Garoff [4] another definition is discussed of wetting hysteresis, the straight 
contact line (SCL) approximation defined as

$$
\begin{aligned}
\cos \theta^{a, S C L} & =\max _{0 \leqslant z<\lambda_{z}} \frac{1}{\lambda_{y}} \int_{0}^{\lambda_{y}} \cos \theta(y, z) d y, \\
\cos \theta^{r, S C L} & =\min _{0 \leqslant z<\lambda_{z}} \frac{1}{\lambda_{y}} \int_{0}^{\lambda_{y}} \cos \theta(y, z) d y,
\end{aligned}
$$

where $\theta(y, z)$ is given by Eq. (4).

We suggest here a different definition (more specifically, a way of averaging) that has the advantage of being applicable in both the static and dynamic cases (in the limiting case of small capillary and Reynolds numbers), since it is based on our experience in the dynamic case. More precisely, since the static advancing and receding contact angles are defined as resulting from the preceding CL motion in the respective direction (caused by the plate motion), let us consider first the dynamic case by introducing the plate velocity $u$. The plate velocity is assumed to be positive if the plate motion is directed upward. Then one can write $H=u t$ and express the CL height as $h=h(y, t)$. Experimentally, the dynamic apparent $\mathrm{CA}$ is obtained by averaging over some time $P$,

$$
\cos \theta_{\mathrm{app}}=\frac{1}{\lambda_{y} P} \int_{0}^{P} d t \int_{0}^{\lambda_{y}} \cos \theta(y, h(y, t)) d y .
$$

When the defect pattern is periodic (in the vertical direction), so is the CL dynamics after an initial transient time. The temporal period is $P=\lambda_{z} /|u|$. By performing a change of variables one obtains

$$
\cos \theta_{\mathrm{eff}}=\frac{1}{\lambda_{y} \lambda_{z}} \int_{0}^{\lambda_{z}} d H \int_{0}^{\lambda_{y}} \cos \theta(y, h(y, H)) d y .
$$

A parametric dependence of $h$ on $u$ is assumed here. Such a definition does not contain the time explicitly and may thus be applied also to the static case where $u \rightarrow 0$. This expression is close to the surface averaging of the CA cosine that was used by many authors; see e.g., [24]. By applying this expression to the states $h^{ \pm}$discussed above, one obtains finally the expressions for the advancing and receding CAs:

$$
\begin{aligned}
& \cos \theta^{r}=\frac{1}{\lambda_{y} \lambda_{z}} \int_{0}^{\lambda_{z}} d H \int_{0}^{\lambda_{y}} \cos \theta\left(y, h^{+}(y, H)\right) d y, \\
& \cos \theta^{a}=\frac{1}{\lambda_{y} \lambda_{z}} \int_{0}^{\lambda_{z}} d H \int_{0}^{\lambda_{y}} \cos \theta\left(y, h^{-}(y, H)\right) d y .
\end{aligned}
$$

From Eqs. (13) and (17), it is quite clear that $\theta^{r, *}>\theta^{r}$ and $\theta^{a}<$ $\theta^{a, *}$. We consider the use of expressions (17) more appropriate than that of (13). Indeed, since the static configuration appears necessarily after the CL motion, the advancing and receding contact angles must satisfy the definitions $\theta^{a}=\theta_{\text {eff }}(u \rightarrow-0)$ and $\theta^{r}=\theta_{\text {eff }}(u \rightarrow+0)$ that result in (17). Note that such a "dynamic" definition corresponds to the advancing and receding CAs, calculated in numerical approaches that use dynamic (see, e.g., [25]) or pseudodynamic [9] algorithms. Equation (17) is not the only possible way for defining the ACA and RCA. For example, for horizontal averaging instead of Eq. (10) one can use Eqs. (6) and (7). If one uses different

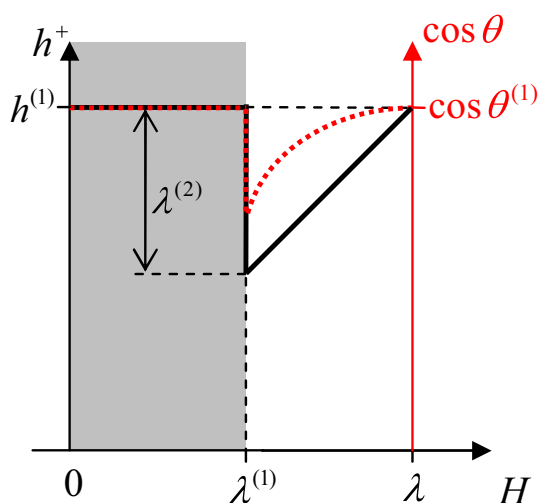

(a)

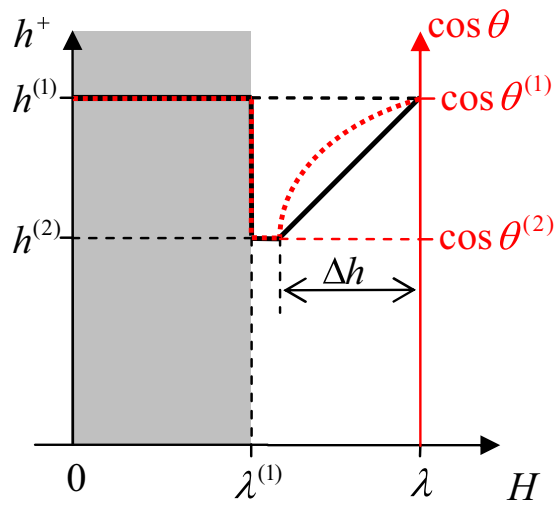

(b)

FIG. 2. (Color online) The highest possible metastable value $h^{+}$ of the CL height (black bold solid line) and the respective CA (red bold dashed line) are shown as functions of the plate position $H$ for the horizontal stripe pattern for one period $\lambda$. This corresponds to the receding case. Two possible situations occur which differ by the relation between $\Delta h$ and $\lambda^{(2)}$ : (a) $\Delta h \geqslant \lambda^{(2)}$ and (b) $\Delta h<\lambda^{(2)}$. A part of the period, where the CL belongs to the domain $\Sigma^{(1)}$, is shaded.

possible averaging also in the vertical direction, then one obtains nine different ways for obtaining the ACAs and RCAs and these all lead to different values of the CA hysteresis.

The importance of the solutions $h^{ \pm}(y, H)$ (for a given plate displacement $H$ ) is not limited to the expressions (17) only. From their definition, it is apparent that the states $h^{ \pm}(y, H)$ may be considered as corresponding to situations, where $H$ varies with time $(H=u t)$ and $u$ approaches zero $[u \rightarrow+0$ for $h^{+}(y, H)$ and $u \rightarrow-0$ for $\left.h^{-}(y, H)\right]$. They present a clear demonstration of the CL stick-slip motion (see, e.g., Figs. 2 and 3 below).

One should note also that the generalization of the expressions (17) to nonperiodic patterns can be obtained by letting both $\lambda_{y}$ and $\lambda_{z}$ go to infinity (in practice, to the respective dimensions of the plate).

\section{HYSTERESIS CALCULATION METHODS}

The free energy functional Eq. (3), as pointed out in the previous sections, depends on the plate vertical position $H$. One needs to obtain the equilibrium meniscus states $f^{ \pm}(x, y)$ that correspond to the CL heights $h^{ \pm}$for each $H \in\left[0, \lambda_{z}\right)$ [this concerns question (ii) in the Introduction]. 


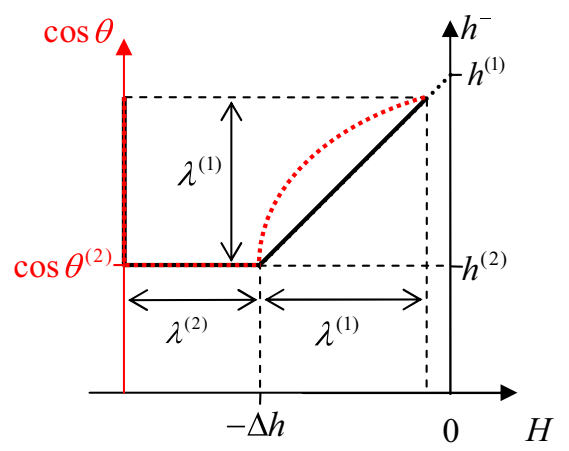

(a)

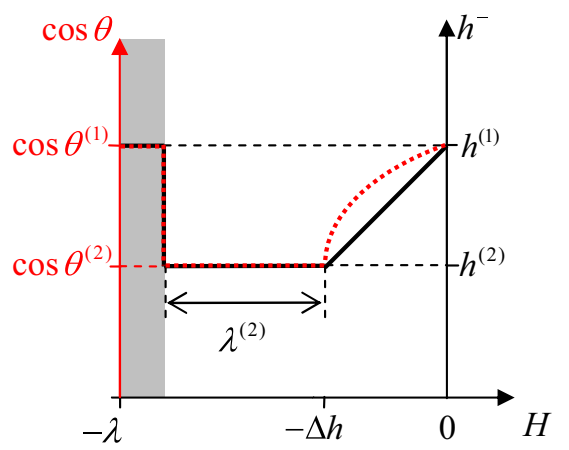

(b)

FIG. 3. (Color online) The lowest possible metastable value $h^{-}$ of the CL height (black bold solid line) and the respective CA (red bold dashed line) are shown as functions of the plate position $H$ for the horizontal stripe pattern for one period $\lambda$. This corresponds to the advancing case. Two situations arise: (a) $\Delta h \geqslant \lambda^{(1)}$ (black slanted dotted line (the dotted line partially coincides with the solid line), a nonperiodic part of the curve $h^{-}(H)$ that appears because of the choice of the plate position corresponding to $H=0$ ) and (b) $\Delta h<\lambda^{(1)}$. A part of the period, where the CL belongs to the domain $\Sigma^{(1)}$, is shaded.

From these solutions, the distributions of the local CAs $\theta\left(y, h^{ \pm}(y, H)\right)$ are found. They are used in Eqs. (17) to find the CA hysteresis.

At fixed $H$, we find the meniscus states $f^{ \pm}(x, y)$ numerically, with an iterative minimization procedure of the energy functional (3). To find the $h^{+}$state that corresponds to the local minimum with the largest $\langle h\rangle$ value, one needs to start iterations from a state with the CL position $h_{0}^{+}(y)$ with $\left\langle h_{0}^{+}\right\rangle \geqslant\left\langle h^{+}\right\rangle$; otherwise, the minimization would bring the system into a wrong local minimum. The opposite inequality should be satisfied for the initial meniscus state $h_{0}^{-}(y)$ used for the "-" state search. The states with the constant CL heights $h^{(1)}$ and $h^{(2)}$, where

$$
h^{(i)}=l_{c} \sqrt{2\left(1-\sin \theta_{\mathrm{eq}}^{(i)}\right)},
$$

may be used for $h_{0}^{+}(y)$ and $h_{0}^{-}(y)$, respectively. Indeed, the above criteria on $h_{0}^{ \pm}$are satisfied because from the conditions (4) and (5) it is evident that for any CL position $h(y)$, $h^{(2)} \leqslant h(y) \leqslant h^{(1)}$. The initial meniscus shapes corresponding to $h^{(i)}$ are obtained with a well known analytical solution of a capillary rise along a partially wetted vertical wall [19].

Every iteration step (let us say, number $k$ ) of the minimization procedure consists of two parts. (i) First, a minimum of the free energy (3) is found for a fixed CL obtained on the iteration $k-1$ with CL height $h_{k-1}=f_{k-1}(0, y)$. This results in a meniscus shape $f_{k}(x, y)$ satisfying the Laplace equation. The resulting local CA distribution $\theta_{k}(\vec{R})$ along the CL $L\left(\vec{R}=\left[y, h_{k}(y)\right]\right)$ may be different from its equilibrium counterpart $\theta(\vec{R})$ given by the conditions (4) and (5).

(ii) In the second part of the iteration step we obtain the new position of the CL height $h_{k}$ by shifting the points of the CL vertically in the direction, decreasing the system free energy. The direction of the CL change, which decreases the system energy, is determined by considering the variation $\delta \vec{R}$ of the point $\vec{R}$ belonging to the CL which leads to the variation of the system energy

$$
\delta U=\gamma_{l g}\left[\cos \theta(\vec{R})-\cos \theta_{k}(\vec{R})\right](\delta \overrightarrow{\vec{R}} z) .
$$

The iterative process stops when there is no movement of the CL that lowers the energy of the system.

In this work we obtain results using two different methods for the first part of the iteration step. The first method (method A) provides the numerical minimization of the energy functional. This is realized as follows. A part of the surface $z=f(x, y)$ defined on a set of width $\lambda_{y}$ and length $d_{x}=10 l_{c}$ is approximated by a set of triangles with $N_{i} \times N_{j}$ node points $\vec{r}_{i j}=\left[x_{i}, y_{j}, f\left(x_{i}, y_{j}\right)\right] ; i=1, \ldots, N_{i} ; j=1, \ldots, N_{j}$. The CL is represented by the points $\vec{r}_{1 j}=\left[0, y_{j}, h\left(y_{j}\right)\right], j=1, \ldots, N_{j}$. The periodic boundary conditions $f\left(x_{i}, y_{1}\right)=f\left(x_{i}, y_{N_{j}}\right), i=$ $1, \ldots, N_{i}$ are imposed in the $y$ direction to simulate periodical solutions. The meniscus shape is determined by the analysis of the variation $\delta U$ of the free energy (3) calculated for small virtual nodal displacements $\delta \vec{r}_{i j}$. The number of points $N_{j}$, which approximate the CL, controls the precision with which the $\mathrm{CL}$ and $\mathrm{CA}$ are determined. High precision of the determination of the CA value is achieved by comparing with solutions obtained with a larger $N_{j}$ value. The set $N_{j}=60,80,100,120$ and the value $N_{i}=1000$ are used. Convergence is assumed to be achieved when the cosine of ACA (or RCA) differs from the respective value obtained for the smaller $N_{j}$ belonging to the above set by less than 0.01 .

The second method (method B) uses an asymptotic solution [4] of the Laplace equation, obtained for small meniscus slopes and arbitrary $h_{k}(y)$ function. In other words, an analytical approximation is used instead of the rigorous numerical minimization. The corresponding local $\mathrm{CA}$ value may be expressed as [22]

$$
\begin{aligned}
\cos \theta_{k}(\vec{R})= & -\frac{1}{2 \lambda_{y}} \sum_{n=-\infty}^{\infty} \sqrt{l_{c}^{-2}+\pi^{2} n^{2} / \lambda_{y}^{2}} \\
& \times \int_{-\lambda_{y}}^{\lambda_{y}} d y^{\prime} h_{k}\left(y^{\prime}\right) \cos \frac{\pi n\left(y-y^{\prime}\right)}{\lambda_{y}} .
\end{aligned}
$$

Instead of using Eq. (19), one finds $\delta h_{k}$ from the pseudodynamic equation

$$
\delta h_{k}(y)=\left[\cos \theta(\vec{R})-\cos \theta_{k}(\vec{R})\right] \delta t,
$$

where $\delta t$ is a small pseudo time step. 
Note that such a theory reduces to that of Joanny and de Gennes [18] in the limit $\lambda_{y} / l_{c} \rightarrow 0$, i.e., for the flat liquid wedge.

\section{RESULTS AND DISCUSSION}

\section{A. Periodic stripe pattern}

The horizontal stripes of different surface energy present the simplest heterogeneity pattern where wetting hysteresis occurs $[9,16]$. The alternating stripes of widths $\lambda^{(1)}, \lambda^{(2)}$, characterized by the CA values $\theta_{\mathrm{eq}}^{(1)}<\theta_{\mathrm{eq}}^{(2)}$, respectively, are considered. The CL heights $h^{ \pm}(H)$ (the $y$ dependence is absent in this case) are periodic functions of period $\lambda_{z}=$ $\lambda^{(1)}+\lambda^{(2)} \equiv \lambda . H=0$ is assumed to correspond to such a plate position, where the CL of height $h=h^{(1)}$ coincides with the stripe border, for which the domain $\Sigma^{(2)}$ is above the domain $\Sigma^{(1)}$. To find $h^{+}(H)$, consider the increase of $H$ that corresponds to the upward displacement of the plate, i.e., the $\mathrm{CL}$ receding. It is evident that the meniscus will remain at equilibrium (i.e., $\theta=\theta_{\mathrm{eq}}^{(1)}$ and $h=h^{(1)}$ ) as long as $H<\lambda^{(1)}$, shown by the solid line in Fig. 2. At $H=\lambda^{(1)}$, the CL slips to a new position with a smaller height and $\theta$ becomes larger. If the stripe $\Sigma^{(2)}$ is wide enough, i.e., $\lambda^{(2)}>\Delta h \equiv h^{(1)}-h^{(2)}$, the CL attains the equilibrium height $h^{(2)}$ during the slip [Fig. 2(b)]. If, on the contrary, $\Delta h \geqslant \lambda^{(2)}$, the CL stops its slip at the domain border [Fig. 2(a)] at the height $h=h^{(1)}-\lambda^{(2)}$ and becomes pinned to that border because the condition (5) of pinning holds. A similar pinning occurs in the case $\lambda^{(2)}>\Delta h$ at $H=\lambda-\Delta h$ (when the CL attains the stripe border). The CL remains pinned while the inequality (5) holds, i.e., while $h \leqslant h^{(1)}$. Note that the CL does not situate at all inside the stripe $\Sigma^{(2)}$ in the case of Fig. 2(a).

The $H$ dependence of the CL height is equivalent to the time dependence introduced as $H=u t$ in the limit $u \rightarrow 0$; cf. Sec. III. For $H$ belonging to regions where $h(H)=h^{(i)}$ (a horizontal line), the meniscus is at equilibrium and CL moves freely inside the stripe $\Sigma^{(i)} ; \theta=\theta_{\mathrm{eq}}^{(i)}$. The CL pinning at a stripe border corresponds to slanted (at $\left.45^{\circ}\right) h(H)$ line portions. The slip corresponds to its vertical portions.

The determination of $h^{-}(H)$ is similar. Again, we start from the same initial position with $h=h^{(1)}$ (Fig. 3). The direction of plate displacement is now downward (advancing CL), and $H<0$. Since $h^{(2)}<h^{(1)}$, the CL remains pinned at the border until its height reaches $h^{(2)}$, which occurs at $H=-\Delta h$. From that moment on, $\theta=\theta_{\mathrm{eq}}^{(2)}$ and $h=h^{(2)}$ as long as $H>-\lambda^{(2)}-$ $\Delta h$. At $H=-\lambda^{(2)}-\Delta h$, CL slips to a new position. If the stripe $\Sigma^{(1)}$ is wide enough $\left(\lambda^{(1)} \geqslant \Delta h\right)$, the equilibrium height $h=h^{(1)}$ and the CA $\theta=\theta_{\mathrm{eq}}^{(1)}$ are both attained [Fig. 3(b)]. In the opposite case [Fig. 3(a)], the CL becomes pinned at the domain border when its height attains $h=\lambda^{(1)}+h^{(2)}<h^{(1)}$ and the new period begins. In this case the CL never again attains its initial height. A part of the $h^{-}(H)$ line between $\lambda^{(1)}+h^{(2)}$ and $h^{(1)}$ does not belong to the periodical curve and for this reason is shown as dotted in Fig. 3(a). Note, that in this case the CL never lies inside the domain $\Sigma^{(1)}$.

The dependence of the cosine of the CA on $H$ can be obtained from the dependence on $H$ of the CL height $h(H)$ by the help of (8). The respective $\cos (\theta)(H)$ dependencies are sketched in Figs. 2 and 3 with red dashed lines.
By comparing Figs. 2 and 3 one can check that the condition $h^{-}(H) \leqslant h^{+}(H)$ is verified (provided both functions are reduced to the same period).

Note, that the simulation from Fig. 2 of David and Neumann [9], where $\lambda^{(1)}=\lambda^{(2)}<\Delta h$, corresponds to the cases of Figs. 2(a) and 3(a).

Consider now the wetting hysteresis. With Eqs. (17), one can obtain by integration the following analytical expressions for RCA and ACA:

$$
\begin{aligned}
\cos \theta^{r}= & \frac{1}{\lambda}\left[\lambda^{(1)} \cos \theta_{\mathrm{eq}}^{(1)}+\max \left(0, \lambda^{(2)}-\Delta h\right)\right. \\
& \times \cos \theta_{\mathrm{eq}}^{(2)}-\frac{l_{c}}{6}\left(2^{3 / 2}\left(1+\sin \theta_{\mathrm{eq}}^{(1)}\right)^{3 / 2}\right. \\
& \left.\left.-\left\{4-\frac{1}{l_{c}^{2}}\left[\max \left(0, \Delta h-\lambda^{(2)}\right)+h^{(2)}\right]^{2}\right\}^{3 / 2}\right)\right], \\
\cos \theta^{a}= & \frac{1}{\lambda}\left[\lambda^{(2)} \cos \theta_{\mathrm{eq}}^{(2)}+\max \left(0, \lambda^{(1)}-\Delta h\right)\right. \\
& \times \cos \theta_{\mathrm{eq}}^{(1)}+\frac{l_{c}}{6}\left(2^{3 / 2}\left(1+\sin \theta_{\mathrm{eq}}^{(2)}\right)^{3 / 2}\right. \\
& \left.\left.-\left\{4-\frac{1}{l_{c}^{2}}\left[\min \left(0, \lambda^{(1)}-\Delta h\right)+h^{(1)}\right]^{2}\right\}^{3 / 2}\right)\right] .
\end{aligned}
$$

Since one has $\left|h^{(i)}\right| \leqslant l_{c} \sqrt{2}$, the above expressions are always real valued. For comparison, the expressions (13) and (14) result in $\theta^{r, S C L}=\theta^{r, *}=\theta_{\text {eq }}^{(1)}$ and $\theta^{a, S C L}=\theta^{a, *}=$ $\theta_{\mathrm{eq}}^{(2)}$ independently of the pattern parameters. These values are independent of the density of the domain boundaries (where, in fact, the pinning occurs).

The advancing and receding CAs calculated with Eqs. (22) as functions of the surface coverage $p^{(2)}=\lambda^{(2)} / \lambda$ by $\Sigma^{(2)}$ stripes are shown in Fig. 4 for the following choice of parameters: $\theta_{\mathrm{eq}}^{(2)}=80^{\circ}, \theta_{\mathrm{eq}}^{(1)}=30^{\circ}$, and $\lambda / l_{c}=0.3,0.1,0.01$. The values of these parameters are the same as in Fig. 8 below. One can see that in the limit $\lambda \rightarrow 0$, the ACA tends to $\theta_{\mathrm{eq}}^{(1)}$ while RCA tends to $\theta_{\mathrm{eq}}^{(2)}$. The spatially averaged (Cassie) CA,

$$
\cos \theta_{C}=p^{(i)} \cos \theta_{\mathrm{eq}}^{(i)}+\left(1-p^{(i)}\right) \cos \theta_{\mathrm{eq}}^{(3-i)},
$$

is also shown on this figure $(i=2)$. It always situates between the receding and the advancing CAs.

Normally, the hysteresis should vanish when the defect coverage tends to zero, i.e., for a homogeneous plate. This corresponds to two cases $p^{(2)} \rightarrow 0\left(\Sigma^{(1)}\right.$-covered plate) and $p^{(2)} \rightarrow 1\left(\Sigma^{(2)}\right.$-covered plate). The hysteresis does not, however, vanish in both these limits in Fig. 4. This happens because the stripe pattern is special. The above limits correspond to the infinitely thin defect stripes. The pinning in this case occurs because the whole CL remains pinned at the defect boundary. This becomes evident if one considers, e.g., Fig. 3 for the 


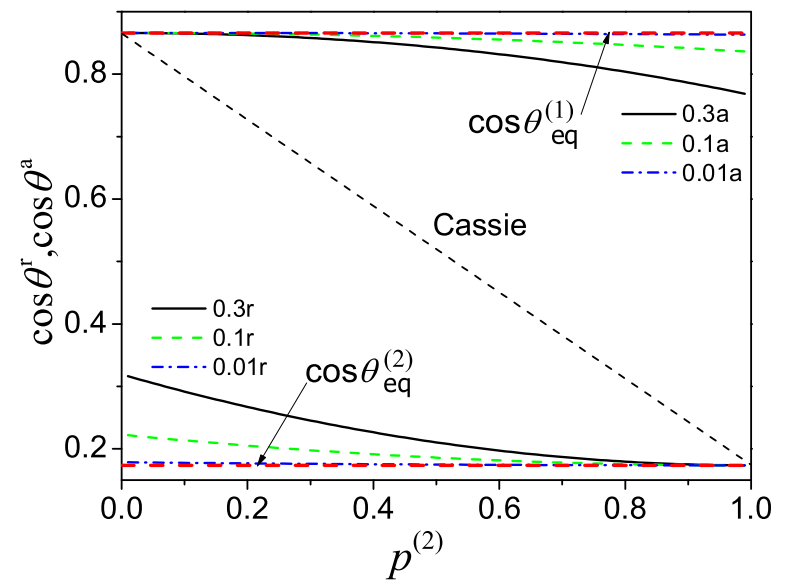

FIG. 4. (Color online) Cosines of the advancing and receding CAs for $\theta_{\mathrm{eq}}^{(2)}=80^{\circ}$ and $\theta_{\mathrm{eq}}^{(1)}=30^{\circ}$ as functions of the surface coverage $p^{(2)}$ by $\Sigma^{(2)}$ stripes for $\lambda / l_{c}=0.3,0.1,0.01$.

advancing case. In the limit $\lambda_{2} \rightarrow 0$ (i.e., $p \rightarrow 0$ ), the CL stick-slip behavior persists. It is shown below that such a pinning does not occur for $y$-dependent defect patterns.

\section{B. Doubly periodic pattern of circular defects on a homogeneous base}

As an example of heterogeneity pattern, where the CA $\theta$ depends not only on the vertical, but also on the horizontal coordinate, a doubly periodic defect pattern (see Fig. 1), studied previously in [22], is considered here. It consists of circles of radius $a$ ordered on a square lattice with a distance $\lambda_{y}=\lambda_{z} \equiv \lambda$ between the centers of two neighboring circles. One of the sides of the square lattice is parallel to the liquid level. For such a pattern, we have chosen the plate position $H=0$ to be the one, where the centers of one of the rows of circular defects coincides with the liquid level far from the plate. From now on all the lengths are considered to be dimensionless, in the units $l_{c}$. In the absence of analytical solution for such a problem, a numerical analysis is performed with the two methods of Sec. IV.

\section{Contact line pinning and hysteresis}

The metastable CL states are studied in this section in the small slope approximation (method B), and for its validation a comparison is made with the method A results. In order to do that, the system parameters are chosen to be the same as in [22]: The circular defects (domains $\Sigma^{(2)}, \theta_{\mathrm{eq}}^{(2)}=110^{\circ}$ ) are less wettable than the rest of the plate (domain $\Sigma^{(1)}$ with $\left.\theta_{\mathrm{eq}}^{(1)}=70^{\circ}\right)$. The period of heterogeneity pattern is $\lambda=0.3$ and the radius of circular defects is $a=0.1$.

First, at a fixed plate position $H$, the meniscus states with the highest and the lowest possible averaged CL heights $h^{ \pm}(y, H)$ are analyzed. During the minimization process, the CL passes through several rows of defects before the equilibrium state is attained.

An example for $h^{+}(y, H)$, obtained by method A, is shown in Figs. 5(a) and 5(c). (for 20 values of $H, H_{i}=i \lambda / 20, i=$ $0,1, \ldots, 19)$.

As mentioned in Sec. IV, the straight CL of height $h=$ $h^{(1)}$ needs to be chosen for the initial state for the iterative

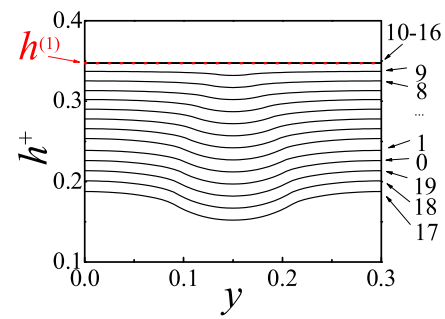

(a)

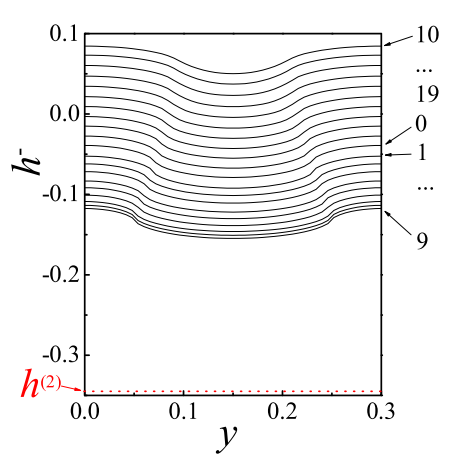

(b)

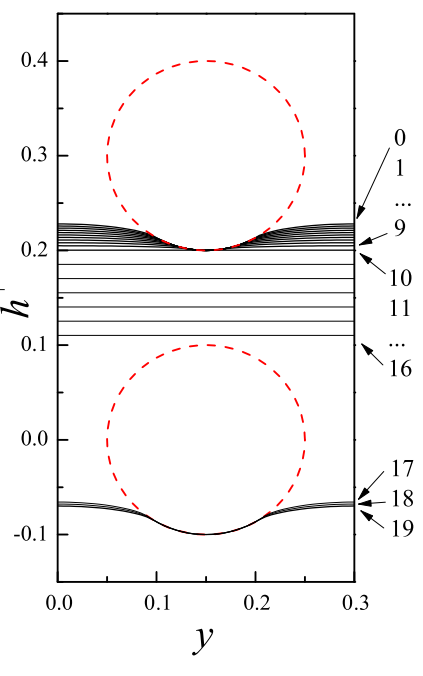

(c)

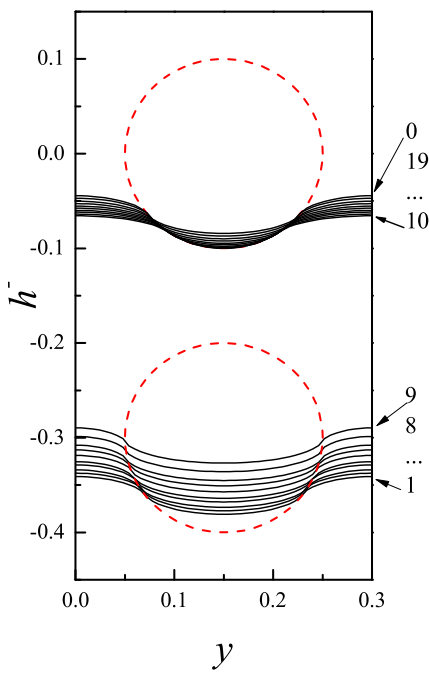

(d)

FIG. 5. (Color online) Contact line shapes $h^{ \pm}\left(y, H_{i}\right)$ calculated for 20 different positions $i=0,1, \ldots, 19$ of the heterogeneity pattern; the index $i$ is the curve parameter. Panels (a) and (c) correspond to $h^{+}\left(y, H_{i}\right)$ with the highest $\langle h\rangle$ (receding case, plate moves upwards, $H_{i}=i \lambda / 20$ ) and (b) and (d) correspond to $h^{-}\left(y, H_{i}\right)$ with the lowest $\langle h\rangle$ (advancing case, plate moves downwards, $H_{i}=-i \lambda / 20$ ). The CLs in panels (a) and (b) are in the reference system of the liquid pool. The CLs in panels (c) and (d) are in the reference system of the plate; however, in panel (c) every CL height $h_{i}$ (and the respective defects) is shifted by $-H_{i}$ and in panel (d) by $H_{i}$. The defect borders (dashed lines) are shown too. 


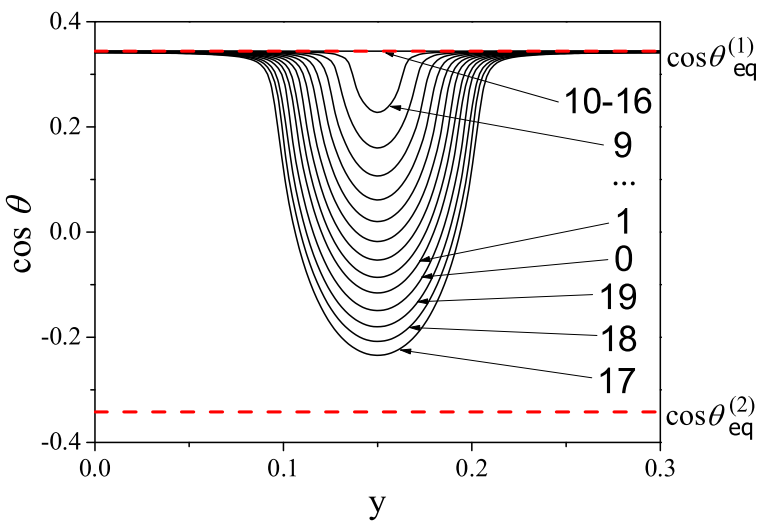

FIG. 6. (Color online) Variation of the local CA along the CL for the receding CL shapes shown in Figs. 5(a) and 5(c).

procedure for every $H$ to find $h^{+}(y, H)$. Since the CL height at the circular spots should be lower than outside them, the CL starts to slide down during the iterations until the equilibrium state is reached. The CL reaches the lower border of the defect and sticks to it. This situation is common for several heights $H_{i}, \quad i=0, \ldots, 9$ (where $\left.H_{i}=i \lambda / 20\right)$, since a range of CAs is allowed at the border of defect according to the condition (5); see Fig. 6. After slip of the CL to the next range of defects, the same behavior is observed for $i=17,18,19$. When the position of heterogeneity pattern with respect to the liquid level is such that the initial CL belongs entirely to the domain $\Sigma^{(1)}$, the initial CL is in equilibrium and $\theta\left(y, H_{i}\right)=\theta_{\mathrm{eq}}^{(1)}$. This is the case for $i=10, \ldots, 16$.

The situation is different for the states with the lowest average height $h^{-}\left(y, H_{i}\right)$ shown in Figs. 5(b) and 5(d) (here $\left.H_{i}=-i \lambda / 20\right)$. During the minimization process, the parts of the initial CL (chosen to be of height $h=h^{(2)}$ ) lying outside the circular defects move toward the height $h^{(1)}$ and reach $\theta=\theta_{\mathrm{eq}}^{(1)}$. At the same time, the parts of the CL inside defects maintain the CA $\theta=\theta_{\mathrm{eq}}^{(2)}$. Unlike the $h^{+}$case, there are no parts of CL pinned at the border of the defects; a part of the CL lies inside defects.

The spatial variation (along the $y$ axis) of $\theta$ reflects the above described CL behavior. In the $h^{-}$(advancing) case, a part of the CL always lies inside defects so that $\theta$ sharply varies at their borders from $\theta_{\mathrm{eq}}^{(1)}$ to $\theta_{\mathrm{eq}}^{(2)}$. In the $h^{+}$(receding) case, a part of CL is pinned on the defect borders and $\theta$ varies smoothly according to (5). Its variation is shown in Fig. 6 for one period $\lambda$ of the surface heterogeneity for the CL shapes corresponding to Figs. 5(b) and 5(d).

With the expression (12), one can obtain the averaged cosines of the CAs. These quantities are shown in Fig. 7 as functions of the plate vertical position $H$ with respect to the liquid level. The results of the small-slope approximation are shown also in the figure with lines: the solid lines for the receding and the dashed lines for the advancing. The symbols show the numerical results by method A: circles for the advancing CAs and squares for the receding CAs.

The numerical results, obtained by method A, are compared to those of the method B. One can see that the agreement is very good.

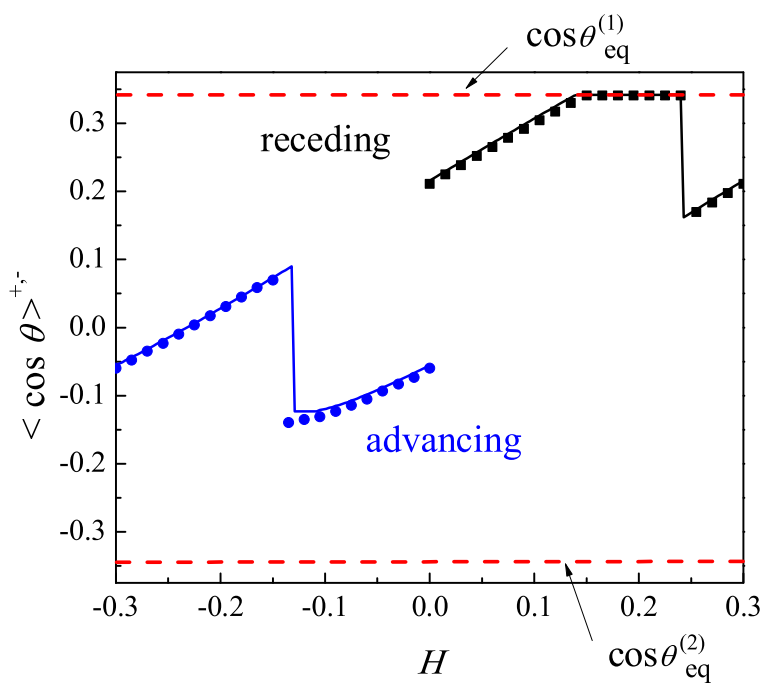

FIG. 7. (Color online) Horizontally averaged cosines of CAs corresponding to the receding and advancing cases. The lines come from the small-slope approximation $(\operatorname{method} B)$; the symbols show the method A results. Each symbol corresponds to a curve in Figs. 5. The numbering is left to right for the receding and right to left for the advancing curve.

Note that, similarly to Figs. 2 and 3, Fig. 7 shows the variation of $\langle\cos \theta\rangle$ for advancing and receding motion (where $H$ should be associated with $u t$ ) in the limit $u \rightarrow 0$. One can compare the behavior for the stripe and the circle patterns. The receding curve in Fig. 7 is directly comparable with Fig. 2(a), however, with a shift caused by the different choices of the $H=0$ position. Since the circle diameter is similar to $\lambda^{(2)}$ of the stripe pattern and the CL initial height in the circle pattern is about $h^{(1)} \approx \lambda+0.5 a$, the curves in Fig. 2(a) should be shifted to the left by about $\lambda^{(1)}+0.25 \lambda^{(2)}$ to be compared. Such a shift makes both cases look completely similar. When $H$ increases, first pinning (corresponding to the slanted parts) occurs both in the receding curve of Fig. 7 and in Fig. 2(a). The pinning corresponds to the curves 0-9 in Figs. 5(a) and 5(c). Then the CL becomes straight in Figs. 5(a) and (5 c), which corresponds to the horizontal parts of both the receding curve in Fig. 7 and Fig. 2(a). Slip (corresponding to the vertical parts) occurs in Fig. 7 and in shifted Fig. 2(a) almost at the same position.

For the advancing case, the difference between the stripe and circle patterns is larger. Similarly to the receding case, the curves of Fig. 3(a) should be shifted by about $0.75 \lambda^{(2)}$ to the right. The advancing curve in Fig. 7 is quite different from that in Fig. 3(a). In the circle case, $\langle\cos \theta\rangle^{-}>\cos \theta^{(2)}$ during the whole period. In other words, the CL never becomes straight [cf. Figs. 5(b) and 5(d)] and no horizontal portion in the $\langle\cos \theta\rangle^{-}(H)$ curve exists. This occurs because there is no possibility for a straight CL to situate entirely inside the $\Sigma^{(2)}$ domain.

From Figs. 5(c) and 5(d), one can deduce that the pinning occurrence at the domain border is independent of the direction of the CL motion and depends only on the side of the border at which the liquid situates. Indeed, the pinning for both advancing and receding motion occurs at a border where the liquid situates at the more wettable side. 
One can check in Fig. 7 that the inequalities $\cos \theta^{(2)} \leqslant$ $\langle\cos \theta\rangle^{-} \leqslant\langle\cos \theta\rangle^{+} \leqslant \cos \theta^{(1)}$ are satisfied, as they should be according to the definition of the " \pm " states.

One needs to note that for all meniscus shapes, the average cosine of the CA [Eq. (11)] coincides with high precision with the cosine of the average CA, obtained by the averaged height of the CL [Eq. (7)], the difference between the two values being less than 0.01 .

The wetting hysteresis interval $\left[\theta^{r}, \theta^{a}\right]=\left[73.58^{\circ}, 92.7^{\circ}\right]$ given by Eqs. (17) can now be compared to that given by Eq. (13) $\left[\theta^{r, *}, \theta^{a, *}\right]=\left[70^{\circ}, 96.5^{\circ}\right]$. The SCL approximation (14) results in $\left[\theta^{r, S C L}, \theta^{a, S C L}\right]=\left[70^{\circ}, 90^{\circ}\right]$. One can see that the hysteresis values given by Schwartz and Garoff [4] are considerably off those obtained with Eqs. (17) compatible with the dynamics history of the CL motion. All the above numbers have been obtained with method A of Sec. IV. On the other hand, the accuracy of the small-slope approximation (method B) results in $\left[\theta^{r}, \theta^{a}\right]=\left[73.6^{\circ}, 91.2^{\circ}\right]$. These data provide a good accuracy: The hysteresis is within $2^{\circ}$ from the method A results.

\section{Wetting hysteresis parametric analysis}

In this section we discuss the dependence of the wetting hysteresis magnitude on the surface coverage of the plate by the defects for the circle pattern and compare it to the stripe pattern; cf. Fig. 4. We take advantage of the possibility to calculate with the method A the menisci of arbitrary (high) slopes and use the values $\theta_{\mathrm{eq}}^{(2)}=80^{\circ}$ and $\theta_{\mathrm{eq}}^{(1)}=30^{\circ}$ so that the results are comparable with those of the previous works [9,10]. Similarly to the previous section, the case of less wettable defects on more wettable base is considered first. The surface coverage is defined in this case as $p^{(2)}=\pi a^{2} / \lambda^{2}$. The calculations have been performed for $p^{(2)}<0.75$ : For $a>\lambda / 2(p>\pi / 4)$ the circles overlap and the pattern topology changes.

The cosines of the advancing and receding CAs calculated with Eq. (17) for both patterns are shown in Fig. 8 for different but fixed $\lambda$ values and varying $a$. The tendency is similar

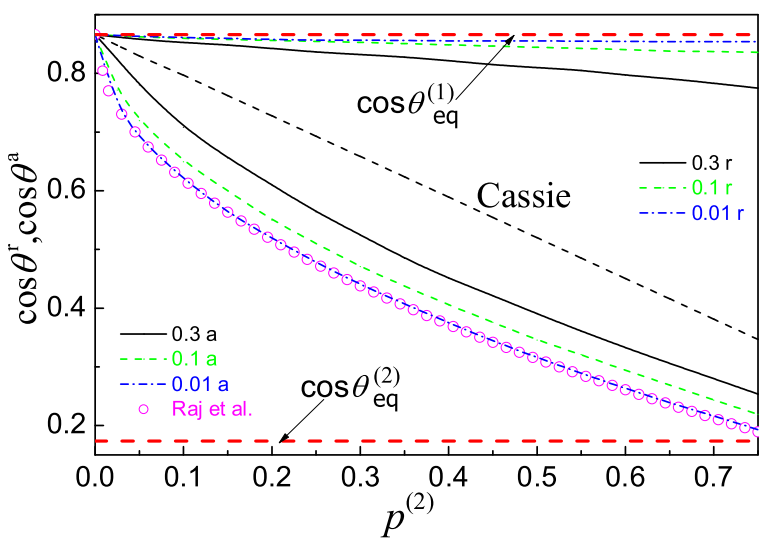

FIG. 8. (Color online) Cosines of the advancing and receding CAs for the doubly periodic pattern of circular defects as functions of the defect surface coverage for fixed values of the spatial period $\lambda=0.3,0.1,0.01$. The equilibrium CA is $\theta_{\mathrm{eq}}^{(2)}=80^{\circ}$ on the circles and $\theta_{\mathrm{eq}}^{(1)}=30^{\circ}$ on the base. The prediction of Raj et al. [17] is also shown with open circles. at fixed $a$ and varying $\lambda$. For a more complete picture, the Cassie"s angle (23) $(i=2)$ is also shown on this figure with a dotted line.

The RCA value remains close to $\theta_{\mathrm{eq}}^{(1)}$ and the cosine of the ACA decreases with $p^{(2)}$. As a result, the hysteresis amplitude increases with the surface coverage, which agrees qualitatively with the experimental and other numerical [10,25-27] data. The difference of the cosines of the receding and advancing CAs obtained by the help of Eqs. (9) and (10) is not a linear or quadratic function of the defect concentration. This results is in contrast to the case of randomly heterogeneous surfaces, where we obtain quadratic dependence on the concentration of defects [10].

Note that the defect coverage $p^{(2)}$ cannot be considered as a unique parameter, characterizing hysteresis; the hysteresis depends also on the heterogeneity period $\lambda$. It is, however, likely that the latter dependence will disappear in the limit $\lambda \rightarrow 0$, i.e., for periods of the heterogeneity patterns much smaller than the macroscopic liquid surface curvature. This tendency is suggested by the expression (22) for the cosine of the local CA that saturates in the limit $\lambda / l_{c} \rightarrow 0$.

At this stage, a comparison can be performed with the semiempirical model of Raj et al. [17]. This model considers the CL behavior for the same circle pattern. Raj et al. proposed that the receding CL stays always between the less wettable defects, which corresponds to $\theta^{r \text {,Raj }}=\theta_{\text {eq }}^{(1)}$. As for the advancing CL, it was argued to belong partly (on average, the CL length $2 a$ per period $\lambda$ ) to the defects. This results in [note that there are typos in Eq. (8) of Raj et al. [17] that is supposed to correspond to their Fig. 5(b); numbers 1 and 2 should be interchanged]

$$
\cos \theta^{a, \text { Raj }}=\frac{2 a}{\lambda} \cos \theta_{\mathrm{eq}}^{(2)}+\left(1-\frac{2 a}{\lambda}\right) \cos \theta_{\mathrm{eq}}^{(1)} .
$$

This result is shown with open circles in Fig. 8. One can see that Eq. (24) agrees with the rigorous numerical data in the limit $\lambda \rightarrow 0$ [the model (24) is independent of this parameter]. The same tendency was obtained [10] for the case of square shaped defects.

The above data are obtained for less wettable defects on a more wettable base. One can consider the opposite case of the domain $\Sigma^{(1)}$ formed by the circular defects with $\theta_{\mathrm{eq}}^{(1)}=30^{\circ}$ on the base $\Sigma^{(2)}$ with $\theta_{\mathrm{eq}}^{(2)}=80^{\circ}$. The defect surface coverage in this case is $p^{(1)}=\pi a^{2} / \lambda^{2}$. The ACA and RCA results are inverted with respect to the former case. The ACA remains close to $\theta_{\mathrm{eq}}^{(2)}$, while the cosine of RCA grows with $p^{(1)}$. The hysteresis vanishes in the limit $p^{(1)} \rightarrow 0$. The Cassie's angle, given by Eq. (23) with $i=1$, situates again between the ACA and RCA. An example of the calculated RCA variation with $p^{(1)}$ for $\lambda=0.03$ is shown in Fig. 9. Raj et al. [17] provide the following expression for this case:

$$
\cos \theta^{r, \text { Raj }}=\frac{2 a}{\lambda} \cos \theta_{\mathrm{eq}}^{(1)}+\left(1-\frac{2 a}{\lambda}\right) \cos \theta_{\mathrm{eq}}^{(2)} .
$$

This dependence is also shown in Fig. 9. The agreement with the numerical data, obtained for a small $\lambda$, is good. 


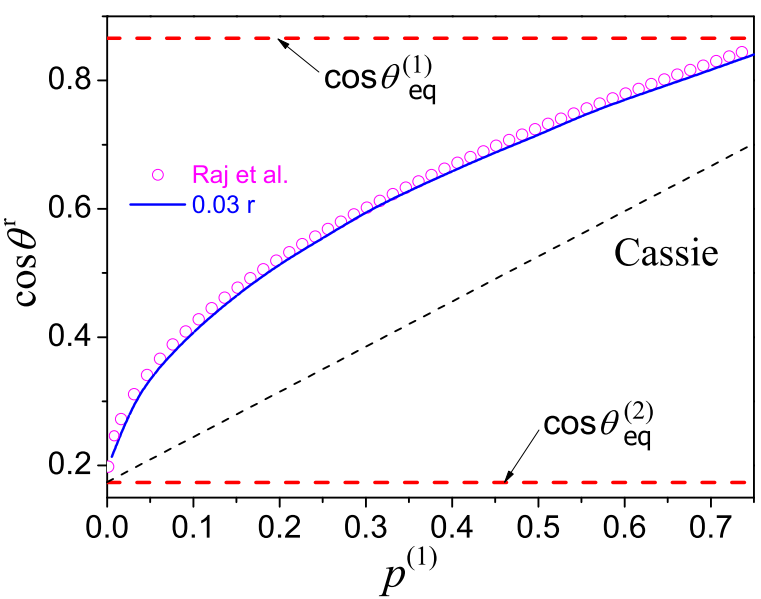

FIG. 9. (Color online) Cosine of the RCA as function of the surface coverage, calculated for $\lambda=0.03$ for the doubly periodic pattern of circular defects with more wettable circles with $\theta_{\text {eq }}^{(1)}=30^{\circ}$ and less wettable base with $\theta_{\mathrm{eq}}^{(2)}=80^{\circ}$. The prediction of Raj et al. [17] is also shown with open circles.

\section{CONCLUSION}

In this work we suggest a general approach for determination of the advancing and receding contact angles and discuss how to find them from known properties of the substrate heterogeneity. Our expressions for the static angles account for the preceding CL motion history and result in the values measurable with the Wilhelmy balance. We have shown that when determining the ACA and RCA it matters how one averages the CA, first at fixed position of the plate pattern with respect to the liquid level and second over the many different possible relative positions of the defect pattern on the plate with respect to the liquid meniscus.

Two examples of periodic heterogeneity patterns are used to illustrate the wetting hysteresis determination. One of them is the simplest pattern in which the hysteresis occurs, the alternating horizontal stripes of different wettability. The analytical expressions for the advancing and receding contact angles are obtained for it. The analysis of the other, doubly periodic pattern of circular defects, has been carried out by methods A and B. Their comparison shows the validity of the small-slope approximation that needs much less computation time. A comparison with other existing expressions for the wetting hysteresis has also been carried out. It is confirmed that the determination [10] of the CA hysteresis using the averaged CL height or through the averaged cosine of the CA lead to the same results.

The pinning on both the stripe and the doubly periodic patterns has been discussed in some detail. Their similarities and differences have been revealed. It has been shown that the pinning occurrence at a border between the domains of different wettability is independent of the direction of the CL motion. The pinning occurs at the border where the liquid situates at the more wettable side.

Next, the hysteresis was analyzed as a function of the defect density. This function also differs from the dependence, found for the randomly distributed defects, and is close to the dependence, found for the square checkerboard defect pattern. It was shown that for the case when the pattern period is much smaller than the average liquid meniscus radius of curvature, the wetting hysteresis is well described by the semiempirical expressions of Raj et al. [17].

Cassie's angle is one of the parameters, characterizing the heterogeneous surfaces, however, it is not sufficient for characterizing the heterogeneous surfaces. Our results displayed in Figs. 4, 8, and 9 show that the ACAs and the RCAs [calculated by the help of Eq. (17)] remain different from Cassie's angle (its value is always between the ACA and the RCA). This holds true also in the limit of minuscule defects. We note here again that these results are valid for heterogeneous surfaces with abrupt changes of the surface tension.

Although the definitions for RCA and ACA were studied on the example of two heterogeneity patterns, there is no limitation for studying the rough surfaces in Cassie's regime. The application to this case is straightforward. However, the application of this approach to studying CA hysteresis on rough surfaces in Wentzel's regime, as well as the application of the same approach for studying the wetting hysteresis in dynamics, needs further development and will be a subject of future research.
[1] D. Bonn, J. Eggers, J. Indekeu, J. Meunier, and E. Rolley, Rev. Mod. Phys. 81, 739 (2009).

[2] P.-G. de Gennes, Rev. Mod. Phys. 57, 827 (1985).

[3] M. Järn, Q. Xu, and M. Lindén, Langmuir 26, 11330 (2010).

[4] L. W. Schwartz and S. Garoff, Langmuir 1, 219 (1985).

[5] S. Brandon and A. Marmur, J. Colloid Interface Sci. 183, 351 (1996).

[6] S. D. Iliev and N. C. Pesheva, Langmuir 19, 9923 (2003).

[7] J. Long, M. N. Hyder, R. Y. M. Huang, and P. Chen, Adv. Colloid Interface Sci. 118, 173 (2005).

[8] M. A. Rodríguez-Valverde, F. J. M. Ruiz-Cabello, and M. A. Cabrerizo-Vilchez, Adv. Colloid Interface Sci. 138, 84 (2008).

[9] R. David and A. W. Neumann, Langmuir 26, 13256 (2010).

[10] D. Iliev, N. Pesheva, and S. Iliev, Langmuir 29, 5781 (2013).
[11] H. Kusumaatmaja and J. M. Yeomans, Langmuir 23, 6019 (2007).

[12] S. Vedantam and M. V. Panchagnula, Phys. Rev. Lett. 99, 176102 (2007).

[13] W. Jin, J. Koplik, and J. R. Banavar, Phys. Rev. Lett. 78, 1520 (1997).

[14] R. J. Good, J. Am. Chem. Soc. 74, 5041 (1952).

[15] R. E. Johnson and R. H. Dettre, J. Phys. Chem. 68, 1744 (1964).

[16] A. W. Neumann and R. J. Good, J. Colloid Interface Sci. 38, 341 (1972)

[17] R. Raj, R. Enright, Y. Zhu, S. Adera, and E. N. Wang, Langmuir 28, 15777 (2012).

[18] J. F. Joanny and P. G. de Gennes, J. Chem. Phys. 81, 552 (1984). 
[19] L. D. Landau and E. M. Lifshitz, Fluid Mechanics (Pergamon Press, Oxford, 1987).

[20] S. Brandon, N. Haimovich, E. Yeger, and A. Marmur, J. Colloid Interface Sci. 263, 237 (2003).

[21] S. Moulinet, C. Guthmann, and E. Rolley, Eur. Phys. J. B 37, 127 (2004)

[22] V. S. Nikolayev, J. Phys. Condens. Matter 17, 2111 (2005).
[23] E. B. Dussan V. and R. T.-P. Chow, J. Fluid Mech. 137, 1 (1983).

[24] P. S. Swain and R. Lipowsky, Langmuir 14, 6772 (1998).

[25] B. M. Mognetti and J. M. Yeomans, Langmuir 26, 18162 (2010).

[26] M. Reyssat and D. Quéré, J. Phys. Chem. B 113, 3906 (2009).

[27] A. L. Dubov, J. Teisseire, and E. Barthel, Europhys. Lett. 97, 26003 (2012). 